\title{
Is There Evidence of Early Vascular Disease in Patients with Obstructive Sleep Apnoea Without Known Comorbidities? Preliminary Findings
}

\author{
P. Steiropoulos ${ }^{1, *}$, C. Bogiatzi ${ }^{2}$, K. Archontogeorgis ${ }^{1}$, E. Nena ${ }^{1}$, M. Xanthoudaki ${ }^{1}$, P. Boglou ${ }^{1}$, A. \\ Tzouvelekis ${ }^{1}$, N. Papanas ${ }^{3}$, G. Tsivgoulis ${ }^{2,4}$ and D. Bouros ${ }^{1}$ \\ ${ }^{1}$ Department of Pneumonology, Medical School, Democritus University of Thrace, Alexandroupolis, Greece \\ ${ }^{2}$ Department of Neurology, Medical School, Democritus University of Thrace, Alexandroupolis, Greece \\ ${ }^{3}$ Second Department of Internal Medicine, Medical School, Democritus University of Thrace, Alexandroupolis, Greece \\ ${ }^{4}$ International Clinical Research Center, St. Anne's University Hospital, Brno, Czech Republic
}

\begin{abstract}
We evaluated early atherosclerotic lesions in 20 non-smokers with newly diagnosed Obstructive Sleep Apnoea (OSA) and without known comorbidities by measuring common carotid artery intima media thickness (CCA-IMT), transcranial Doppler ultrasound (TCD), and ankle brachial index (ABI). These were compared with 20 healthy age- and BMImatched controls. In OSA patients, CCA-IMT was not significantly higher vs. controls $(0.74 \pm 0.17$ vs. $0.66 \pm 0.12 \mathrm{~mm}$, $\mathrm{p}=0.201)$ and it was positively correlated with neck circumference $(\mathrm{r}=0.466, \mathrm{p}=0.039)$, arousal index $(\mathrm{r}=0.663, \mathrm{p}=0.001)$, gamma-glutamyl transpeptidase activity $(\mathrm{r}=0.474, \mathrm{p}=0.035)$ while it was negatively correlated with Forced Expiratory Volume in $1 \mathrm{sec}(\mathrm{r}=-0.055, \mathrm{p}=0.012)$. No difference was noted between patients and controls in terms of vascular stenosis on TCD examination, while asymptomatic peripheral artery disease was found in one patient with OSA. In conclusion, OSA patients without known comorbidities exhibit a non-significant increase in CCA-IMT without further evidence of vascular disease, but additional experience in a larger patient series is needed.
\end{abstract}

Keywords: Ankle-Brachial Index, Atherosclerosis, Intima-Media Thickness, Obstructive Sleep Apnoea, Transcranial Doppler, Vascular disease.

\section{INTRODUCTION}

Obstructive Sleep Apnoea (OSA) is associated with increased risk for cardiovascular disease (CVD) [1-3] and cerebrovascular morbidity $[4,5]$. Oxygen desaturation accompanying apneic events, negative intra-thoracic pressure, arousals induced by upper airway obstruction, and repeated activation of the sympathetic system could cause an abnormal activation of neural, humoural, thrombotic, metabolic and inflammatory responses, thereby promoting atherosclerosis [6-8].

Common Carotid Artery Intima-Media Thickness (CCAIMT) is a useful parameter to evaluate the severity of early atherosclerosis $[9,10]$ and the overall vascular disease risk $[11,12]$. Several studies have documented a link between increased CCA-IMT and OSA [13-16]. Transcranial Doppler ultrasound (TCD) is a simple non-invasive procedure that investigates intracranial flow with multiple indications in several clinical conditions [17]. The Ankle-Brachial-Index (ABI) is a practical, widely used and reliable diagnostic tool for peripheral arterial disease (PAD); it is also useful for the screening of CVD $[18,19]$.

*Address correspondence to this author at the Sleep Unit, Department of Pneumonology, Medical School, Democritus University of Thrace, 68100 Alexandroupolis, Greece; Tel: +302551075333;

Fax:+302551076106; E-mail: pstirop@med.duth.gr
This preliminary case-series study aimed to examine early atherosclerotic lesions in patients with recently diagnosed OSA free from other known comorbidities. Early vascular lesions were assessed by CCA-IMT, ABI and TCD. To our knowledge, this is the first study that combines CCAIMT, TCD and ABI measurements for the evaluation of vascular disease in OSA patients.

\section{MATERIALS AND METHODOLOGY}

\section{PATIENTS}

The study included 20 patients (16 males and 4 females) referred to our sleep laboratory and 20 healthy subjects (16 males and 4 females) who were used as controls. All subjects were non-smokers and underwent laboratory and clinical examination. Information regarding previous medical history, tobacco smoking and alcohol consumption was obtained. Height, weight, body-mass index (BMI) [BMI = weight $(\mathrm{kg}) /$ height $\left.^{2}(\mathrm{~m})\right]$, neck circumference, hip circumference, waist circumference and waist/hip ratio were measured using a standardised protocol [20]. Briefly, neck circumference was calculated at the cricothyroid level, waist circumference in the midpoint between the 12th rib and the iliac crest, and hip circumference was measured at the level of the greater trochanter. 
Electrocardiogram and arterial blood pressure (BP) measurement were performed. Arterial blood gases were analysed. Venous blood samples were obtained the morning after polysomnography (PSG) after $8 \mathrm{~h}$ fasting, during which only water intake was permitted. Levels of total cholesterol, triglycerides, high- and low-density lipoprotein were measured by enzymatic colorimetric methods, fasting glucose was determined by enzymatic method, while C-reactive protein, gamma-glutamyl transferase (GGT), aspartate aminotransferase (AST) and alanine aminotransferase (ALT) were measured by an immunoturbidimetric method (Olympus AU640 ${ }^{\mathrm{TM}}$, Olympus Diagnostica GmbH, Hamburg, Germany). Pulmonary function tests were performed as well (Screenmate, Erich Jaeger GmbH \& Co., Hochberg, Germany).

Somnolence was evaluated by the validated Greek version of the Epworth Sleepiness Scale (ESS) [21], a selfadministered questionnaire estimating the risk of falling asleep in a variety of situations [maximum score: 24; score $>$ 10: excessive daytime sleepiness (EDS)] [22].

Diabetes was defined as fasting glucose levels $\geq 126$ $\mathrm{mg} / \mathrm{dL}$ or glycated haemoglobin $(\mathrm{HbAlc}) \geq 6.5 \%$ [23]. Arte- rial hypertension was defined as: either systolic $\mathrm{BP}>140$ $\mathrm{mmHg}$ and/or diastolic BP $>90 \mathrm{mmHg}$, both measured on 3 separate occasions, or medical history of documented high arterial BP and use of antihypertensive treatment [24]. Exclusion criteria were as follows: mixed or central apnoeas, chronic obstructive pulmonary disease, cardiac failure or myocardial infarction, kidney disease, cerebrovascular disease, cancer, various causes of daytime sleepiness and any psychiatric or neurological disorder or any other acute medical condition.

All subjects gave their informed consent and the study was approved by the institutional ethics committee.

\section{CCA-IMT}

CCA-IMT was assessed by high-resolution B-mode ultrasonography, as previously described [25]. The left and right common carotid arteries were examined in the anterolateral, posterolateral and mediolateral directions. All subjects were examined in the supine position, with the head turned $45^{\circ}$ from the site being scanned. Both carotid arteries were scanned longitudinally to visualise the IMT in the distal wall of the artery. The best images of the distal wall were

Table 1. Anthropometric, Clinical and Laboratory Measurements of OSA Patients

\begin{tabular}{|l|l|}
\hline Variables & $\begin{array}{l}\text { Mean } \pm \text { standard deviation } \\
\text { (Range) }\end{array}$ \\
\hline Age (years) & $50.8 \pm 12.4(28-72)$ \\
\hline Males/females & $16 / 4$ \\
\hline Body weight (kg) & $101.8 \pm 20.6(57-139)$ \\
\hline BMI (kg/m $\left.{ }^{2}\right)$ & $34.3 \pm 7.2(24.8-50.4)$ \\
\hline Alcohol (drinks/week) & $1.20 \pm 1.00(0-3)$ \\
\hline Neck circumference (cm) & $42.6 \pm 4.1(35-50)$ \\
\hline Waist circumference (cm) & $118.2 \pm 13.1(97-137)$ \\
\hline Hip circumference (cm) & $114.7 \pm 10.3(101-142)$ \\
\hline Waist to hip ratio & $1.03 \pm 0.06(0.9-1.2)$ \\
\hline Total cholesterol (mg/dl) & $202 \pm 46(133-298)$ \\
\hline LDL-cholesterol (mg/dl) & $116 \pm 44(27-183)$ \\
\hline HDL-cholesterol (mg/dl) & $46 \pm 9(33-60)$ \\
\hline Triglycerides (mg/dl) & $202 \pm 172(65-856)$ \\
\hline Glucose (mg/dl) & $95 \pm 10(80-116)$ \\
\hline GGT (mg/dl) & $33 \pm 24(2-101)$ \\
\hline CRP (mg/dl) & $0.46 \pm 0.51(0.08-1.82)$ \\
\hline Systolic blood pressure (mmHg) & $119.5 \pm 9.0(100-135)$ \\
\hline Diastolic blood pressure (mmHg) & $76.0 \pm 6.8(60-85)$ \\
\hline FEV (\% predicted) & $91.0 \pm 19.9(54-132)$ \\
\hline FVC (\% predicted) & $87.5 \pm 20.3(47-131)$ \\
\hline Abbrevis $)$ \\
\hline (m)
\end{tabular}

Abbreviations: BMI, Body Mass Index; CRP, C-Reactive Protein; $\mathrm{FEV}_{1}$, Forced Expiratory Volume in the first second; FVC, Forced Vital Capacity; GGT, Gamma-Glutamyl Transferase; HDL, High Density Lipoprotein; LDL, Low Density Lipoprotein; OSA, Obstructive Sleep Apnoea. 
used to determine the CCA. Using cine-loop function, an optimal longitudinal freeze-frame image in the end-diastolic state was measured manually by calipers. CCA-IMT was defined as the mean of the right and left IMT of the common carotid artery, calculated from 5 measurements on each side, which were taken $10 \mathrm{~mm}$ proximal to the carotid bifurcation. The lumen/intima leading edge (I line) to media/adventitia leading edge ( $\mathrm{M}$ line) method, which has been previously validated anatomically [26] was used. Subjects with evidence of plaques (defined as a focal structure encroaching into the arterial lumen of at least $0.5 \mathrm{~mm}$ or $50 \%$ of the surrounding IMT value) at the site of CCA-IMT measurements were excluded from further evaluation. The reproducibility of CCA-IMT measurements between and within our sonographers has been previously established [25].

\section{ABI}

On the day before PSG, ABI was measured using a handheld $8 \mathrm{MHz}$ Doppler device (MiniDop EX-100Vx, Hadeco, Japan) [27]. Systolic BP was measured in both the dorsalis pedis and posterior tibial artery, and the higher of these pressures was divided by the higher of the 2 brachial systolic BPs to calculate the ABI. Peripheral artery disease (PAD) was defined as $\mathrm{ABI} \leq 0.90$, while $\mathrm{ABI}>1.0$ was considered normal. Subjects with elevated ABI $(>1.30)$ suggestive of poorly compressible leg arteries [28] were excluded.

\section{PSG}

Overnight PSG (Alice ${ }^{\circledR}$ 4, Philips Respironics, Murrysville, PA, USA) was performed from 22:00 to 06:00 hours, as previously described [29]. A standard montage of electroencephalogram, electro-occulogram, electromyogram and electrocardiogram signals was used. Pulse oximetry was registered and airflow was detected using combined oronasal thermistors. Thoracic cage and abdominal motion were also recorded using inductive plethysmography. Apnoeas, hypopnoeas, and electroencephalogram recordings were manually scored according to standard criteria [30]. Apnoea was defined as complete cessation of airflow for at least $10 \mathrm{sec}$. Hypopnea was defined as a 50\% reduction in airflow for at least $10 \mathrm{sec}$ in combination with oxyhaemoglobin desaturation of at least $4 \%$ or an arousal registered by the electroencephalogram [30]. Apnoea-hypopnea index (AHI) was established by the number of apnoeas and hypopneas per hour of PSG-recorded sleep time [30]. Oxygen desaturation index was defined as the number of oxyhemoglobin desaturations of at least 3\% per hour of PSG-recorded sleep time. OSA was defined as AHI $\geq 5$ accompanied by daytime symptoms [31]. OSA was graded as mild (AHI: 5-15/h), moderate (AHI: $15-30 / h)$, and severe $(\mathrm{AHI}>30 / \mathrm{h})$ [31]. Patients with purely central apnoeas were excluded.

\section{TCD}

TCD was carried out the day before PSG using a standardised protocol by a neurologist with special training and experience in cerebrovascular ultrasound [32-34]. An insonation depth of $45 \mathrm{~mm}$ or more was employed for the identification of proximal (i.e. M1) middle cerebral artery (MCA) flow signals and depths of 30 to $45 \mathrm{~mm}$ for presumed distal MCA flow signals (M2MCA). After the identification of

Table 2. Sleep ESS, CCA-IMT, ABI and TCD Measurements of OSA Patients

\begin{tabular}{|c|c|}
\hline Variables & $\begin{array}{l}\text { Mean } \pm \text { standard deviation } \\
\text { (Range) }\end{array}$ \\
\hline ESS (score) & $12.50 \pm 5.88(2-24)$ \\
\hline AHI (events/hour) & $48.68 \pm 20.99(10.8-78.5)$ \\
\hline $\operatorname{avSpO}_{2}(\%)$ & $90.62 \pm 3.04(81-94)$ \\
\hline $\operatorname{minSpO} \mathrm{O}_{2}(\%)$ & $73.90 \pm 11.12(36-88)$ \\
\hline TST with $\mathrm{SpO}_{2}<90 \%$ (\%TST) & $96.67 \pm 73.63(19.5-276.5)$ \\
\hline Sleep efficiency (\%TST) & $79.38 \pm 13.59(52.6-96.8)$ \\
\hline Stage 1 (\%TST) & $23.95 \pm 15.87(5.9-60.8)$ \\
\hline Stage 2 (\%TST) & $55.99 \pm 21.58(1.6-89.3)$ \\
\hline Stage $3+4(\% T S T)$ & $8.28 \pm 10.53(0-38.9)$ \\
\hline REM (\%TST) & $8.60 \pm 7.24(0-23.2)$ \\
\hline Arousal index & $40.57 \pm 17.27(8.2-75.2)$ \\
\hline Mean CCA-IMT (mm) & $0.74 \pm 0.17(0,48-1.06)$ \\
\hline Right ABI & $1.10 \pm 0.13(0.8-1.3)$ \\
\hline Left ABI & $1.10 \pm 0.14(0.7-1.3)$ \\
\hline TCD (intracranial stenosis \%) & 0.00 \\
\hline
\end{tabular}

Abbreviations: ABI, Ankle-Brachial Index; AHI, Apnoea Hypopnoea Index; avSpO 2 , average oxyhaemoglobin saturation during sleep time; CCA-IMT, Common Carotid Artery Intima Media Thickness; ESS, Epworth Sleepiness Scale; minSpO 2 , minimum oxyhaemoglobin saturation during sleep time; OSA, Obstructive Sleep Apnoea; REM, Rapid Eye Movement; TCD, Transcranial Doppler; TST, Total Sleep Time. 
both proximal M1MCA and proximal A1 anterior cerebral artery (ACA) signals at the depth of approximately $65 \mathrm{~mm}$ (range $58-70 \mathrm{~mm}$ ), the probe was aimed inferiorly and slightly posteriorly and flow signals of the terminal internal carotid artery (TICA) were obtained at a depth of $60-70 \mathrm{~mm}$ [32]. Posterior cerebral artery (PCA) intracranial atherosclerotic disease was detected using an insonation depth of 58-70 $\mathrm{mm}$ with posterior angulation of the probe during transtemporal insonation [32, 33]. An insonation depth of 40-79 mm and of 80-105 mm was used for the identification of vertebral (VA) and basilar artery (BA) steno-occlusive disease, respectively, during transforaminal or suboccipital insonation $[32,33]$.

The following mean flow velocity (MFV) cut-offs were used for identification of $\geq 50 \%$ stenosis according to the SONIA (stroke outcomes and neuroimaging of intracranial atherosclerosis) trial criteria [35]: MCA: MFV $>100 \mathrm{~cm} / \mathrm{sec}$; TICA: MFV $>90 \mathrm{~cm} / \mathrm{sec}$; VA: MFV $>80 \mathrm{~cm} / \mathrm{sec}$; and BA: MFV $>80 \mathrm{~cm} / \mathrm{sec}$. Given that ACA and PCA atherosclerosis were not evaluated in the SONIA trial, we used a set of previously validated criteria for the detection of intracranial atherosclerosis in these vessels $[32,33]$. Primary TCD findings in ACA stenosis $(\geq 50 \%)$ included a focal and significant mean MFV increase (MFV $\geq 80 \mathrm{~cm} / \mathrm{sec}$ and $\geq 30 \%$ difference compared with the contralateral ACA segment) at a depth of $62-75 \mathrm{~mm}$ [32]. Similarly, a cut-off of $>80 \mathrm{~cm} / \mathrm{sec}$ was used for identification of $\geq 50 \%$ PCA stenosis [33]. Subjects with absent temporal windows were excluded.

\section{STATISTICAL ANALYSIS}

Analysis was carried out using SPSS (Statistical Package for Social Sciences, Chicago, Illinois) v.15.0. Normality was assessed by Kolmogorov-Smirnov test. For normally distributed values, descriptive results were expressed as mean \pm SD. Pearson's correlation coefficients were used to examine the association between variables. Analysis of Variance (ANOVA) and Student's t-test were used to examine differences between groups. Reported p-values are two-tailed and significance was defined at $\mathrm{p}<0.05$.

\section{RESULTS}

The study population comprised 32 males and 8 females with age ranging from 28 to 72 years old (mean $50.80 \pm$ 12.37 years old). All subjects were non-smokers. Their anthropometric, clinical and laboratory characteristics are summarised in Table 1 while the results from PSG, ESS, ABI, TCD and CCA-IMT measurements are reported in Table 2. Regarding severity (based on AHI), OSA was mainly moderate-to-severe (1 patient with mild OSA, 5 patients with moderate OSA and 14 patients with severe OSA), while 14 out of 20 patients reported EDS (ESS $>10$ ).

In OSA patients, CCA-IMT was not significantly higher vs. controls $(0.74 \pm 0.17$ vs. $0.66 \pm 0.12 \mathrm{~mm}, \mathrm{p}=0.201)$. No patient exhibited vascular stenosis on TCD, while one patient had asymptomatic PAD $(\mathrm{ABI}=0.7)$.

Mean CCA-IMT in OSA patients was positively correlated with neck circumference $(\mathrm{r}=0.466, \mathrm{p}=0.039)$ and arousal index $(\mathrm{r}=0.663, \mathrm{p}=0.001)$ while it was negatively correlated with $\mathrm{FEV}_{1}$, expressed as percentage of the pre- dicted value $\left(\mathrm{FEV}_{1} \%\right.$ pred $)(\mathrm{r}=-0.551, \mathrm{p}=0.012)$. Additionally, mean CCA-IMT exhibited a significant positive correlation $(\mathrm{r}=0.474, \mathrm{p}=0.035)$ with GGT activity, while no other correlation was observed between CCA-IMT and other biochemical characteristics. Correlations between CCA-IMT and potential predictors for atherosclerosis are reported in Table 3.

\section{DISCUSSION}

The present study has demonstrated a positive correlation between CCA-IMT and arousal index, neck circumference, and GGT as well as a negative correlation with $\mathrm{FEV}_{1}$, in patients with newly diagnosed OSA and without known comorbidities. Conversely, intracranial vascular stenosis (studied by TCD) and PAD (evaluated by ABI) showed no association with any index of OSA.

The relationship between CCA-IMT and OSA has been examined in several previous studies [13-16, 36], even though the authors recognise that common cardiovascular risk factors could represent confounding variables [16]. Suzuki et al. [14] reported that CCA-IMT was related to AHI, duration of oxygen saturation below $90 \%$ and the minimum oxygen saturation. The correlation between OSA-related hypoxemia and CCA-IMT was independently of AHI, indicating that hypoxia could represent an independent risk factor for arterial wall lesions [14]. Importantly, their patients had several comorbidities other than tobacco smoking (47.3\% arterial hypertension, $18 \%$ dyslipidaemia and $17.4 \%$ diabetes mellitus) that can influence the progression of IMT [37-39]. However, after adjustment for confounding factors, the correlation between IMT and severity of OSA remained significant.

Similar findings have been reported by Szabóová et al. [40]. Patients with cardiovascular comorbidities and OSA exhibited higher IMT, as compared with the OSA group alone. Nevertheless, when adjusted for age, IMT was normalised. In another study [41], severity of oxygen desaturation and BP status were the best predictors for CCA-IMT and atherosclerotic plaque formation in 83 OSA patients. In a Chinese study of 52 patients with OSA [42], CCA-IMT was significantly higher in the moderate $(0.81 \pm 0.24 \mathrm{~mm})$ and severe OSA $(0.91 \pm 0.23 \mathrm{~mm})$ subgroups than in the mild OSA $(0.7 \pm 0.17 \mathrm{~mm})$ subgroup. Minoguchi et al. [43] have also observed increased CCA-IMT in patients with moderate to severe $(1.16 \pm 0.05 \mathrm{~mm}, \mathrm{p}<0.003)$ as compared with those with mild OSA $(0.71 \pm 0.03 \mathrm{~mm}, \mathrm{p}<0.0001)$. A similar observation has been reported by Saletu et al. [44]. However, these results are not confirmed by all studies [36].

In our study, a correlation between arousal index and CCA-IMT was observed. This suggests that sleep fragmentation due to intermittent arousals may result in metabolic and inflammatory deregulation, which, in turn, promotes vascular pathology. Of note, Wattanakit et al. [16] found that respiratory disturbance index, and arousal index failed to show a correlation with CCA-IMT after adjustment for additional risk factors for atherosclerosis in 985 subjects from the general population. Importantly, their patients had various risk factors for cardiovascular disease and presented with a mild to moderate OSA with a mean respiratory disturbance index of 8.7 events/h [16]. By contrast, our patients were free from 
Table 3. Correlations between CCA-IMT and Studied Parameters as Predictors for Atherosclerosis in OSA Patients

\begin{tabular}{|c|c|c|}
\hline Variables & $\mathbf{p}$ & $\mathbf{r}$ \\
\hline Age & 0.071 & 0.412 \\
\hline BMI & 0.602 & 0.124 \\
\hline Neck circumference & 0.039 & 0.466 \\
\hline Waist circumference & 0.214 & 0.29 \\
\hline Hip circumference & 0.326 & 0.231 \\
\hline Waist to hip ratio & 0.499 & 0.161 \\
\hline FEV $_{1}$ (\% predicted) & 0.012 & -0.551 \\
\hline FVC (\% predicted) & 0.050 & -0.443 \\
\hline Glucose & 0.558 & 0.139 \\
\hline GGT & 0.035 & 0.474 \\
\hline Cholesterol & 0.528 & 0.150 \\
\hline Triglycerides & 0.155 & 0.330 \\
\hline HDL-cholesterol & 0.822 & 0.054 \\
\hline LDL-cholesterol & 0.633 & -0.114 \\
\hline CRP & 0.684 & 0.097 \\
\hline ESS & 0.733 & 0.081 \\
\hline Sleep efficiency (\%TST) & 0.085 & 0.406 \\
\hline Stage 1 (\%TST) & 0.59 & 0.128 \\
\hline Stage 2 (\%TST) & 0.986 & -0.004 \\
\hline Stage $3+4(\% T S T)$ & 0.17 & -0.32 \\
\hline REM & 0.158 & -0.328 \\
\hline AHI & 0.26 & 0.264 \\
\hline $\operatorname{avSpO}_{2}$ & 0.383 & -0.206 \\
\hline $\operatorname{minSpO_{2}}$ & 0.873 & 0.038 \\
\hline TST with $\mathrm{SpO}_{2}<90 \%$ & 0.44 & 0.183 \\
\hline Arousal index & 0.001 & 0.663 \\
\hline
\end{tabular}

Abbreviations: AHI, Apnoea Hypopnoea Index; $\mathrm{avSpO}_{2}$, average oxyhaemoglobin saturation during sleep time; BMI, Body Mass Index; CCA-IMT, Common Carotid Artery Intima Media Thickness; CRP, C-Reactive Protein; ESS, Epworth Sleepiness Scale; FEV ${ }_{1}$, Forced Expiratory Volume in the first second; FVC, Forced Vital Capacity; GGT, Gamma-Glutamyl Transferase; HDL, High Density Lipoprotein; LDL, Low Density Lipoprotein; minSpO ${ }_{2}$, minimum oxyhaemoglobin saturation during sleep time; OSA, Obstructive Sleep Apnoea; REM, Rapid Eye Movement; TST, Total Sleep Time.

known comorbidities. Taken together, the new and the earlier data indicate some correlation between OSA and CCAIMT, although further information on the most relevant parameters of OSA is still desirable. Of foremost importance appears to be nocturnal hypoxia $[13,14]$. At the same time, caution is needed not to underestimate the role of comorbidities. Indeed, it is known that the latter may influence some vascular risk factors [45].

Sleepiness evaluated by ESS was identified as an independent predictor of CCA-IMT in a pre-polysomnographic multiple regression model of an Austrian study [46], a finding not confirmed in our work. Daily somnolence is a major, but still not omnipresent, feature of OSA [46, 47]. It has been shown to be linked with glucose deregulation [47], and its contribution to associated morbidity merits further attention [47].

In previous works, CCA-IMT has been shown to correlate with several biochemical parameters, such as total cholesterol [14], $\mathrm{HbA}_{1 \mathrm{c}}$ [14, 44], C-reactive protein [43, 46], fibrinogen [46], interleukin-6 (IL-6) [43], interleukin-18 (IL18) $[42,43]$, and urinary 15-F2t-isoprostane [48]. In our study, we noted a correlation between GGT and CCA-IMT. GGT is known to be associated with arterial stiffness in healthy individuals [49]. Additionally, it has been identified 
as a risk factor for all-cause mortality (especially among young subjects) [50], for cardiovascular disease [51, 52] and stroke [52, 53]. Kanbay et al. [54] found that elevated GGT was an independent predictor of CVD in OSA patients, and

the increase in GGT was proportional to OSA severity. Thus, our finding further supports Kanbay's observations [54], suggesting that GGT is a marker of incipient carotid atherosclerosis in OSA.

We found a significant negative correlation between

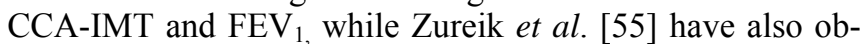
served a negative but insignificant correlation between IMT and $\mathrm{FEV}_{1} \%(\mathrm{r}=-0.13, \mathrm{p}=0.17)$. Additionally, they found that among male subjects, CCA-IMT was higher in those with than those without bronchial hyper-responsiveness $(p=$ 0.002) [55]. This was attributed to the activation of systemic inflammatory mechanisms common to bronchial hyperresponsiveness and arterial injury. In another study [56], CCA-IMT was negatively associated with $\mathrm{FEV}_{1}$, which, in turn, was associated with development of atherosclerotic plaques. In this context, we have provided additional evidence that $\mathrm{FEV}_{1}$ reflects concomitant pulmonary and vascular perturbations. Plausible explanations, other than common inflammatory pathways, may include aging-related physiologic changes affecting both respiratory function and arterial wall thickness, as well as reduced arterial wall oxygenation due to chronic hypoxia.

Several studies assessed the correlation between respiratory function and the risk of stroke. A prospective cohort study of 7151 men with a mean follow up of 14.8 years [57] showed that reduced $\mathrm{FEV}_{1}$ was linked with increased stroke risk even after adjustment for additional CVD risk factors. Men with lower $\mathrm{FEV}_{1}(<3.10$ l) had a $50 \%$ increase in stroke risk compared with those with higher $\mathrm{FEV}_{1}(\geq 3.651)$ with a corresponding relative risk (RR) of 1.4 [57]. Similar results were observed in a Danish study [58]. In another prospective study of 379 women with a mean follow up of 26 years [59], middle- and late-life respiratory function was related to cerebrovascular disease shown by brain computed tomography. For an 1-standard deviation decrease in $\mathrm{FEV}_{1}$, there was increased risk of white matter lesions [odds ratio (OR): 1.46] and lacunar infarcts (OR: 1.42) detected by MRI [59]. In addition, Liao et al. [60] have demonstrated a relationship between lower pulmonary function and subclinical CVD detected by MRI. All this data underlines the importance of our reported association between CCA-IMT and $\mathrm{FEV}_{1}$. It appears, then, that $\mathrm{FEV}_{1}$ is not by chance associated with CCA-IMT but it is, indeed, a risk marker of stroke, and so it should be measured in OSA patients.

This study failed to show a correlation between OSA and TCD. The small patient number may account for this finding. Foster et al. [61] have studied cerebral blood flow in response to hypoxia and the effect of treatment with continuous positive airway pressure (CPAP). Cerebral blood flow in response to isocapnic hypoxia was assessed by TCD in 8 male OSA patients before and after 4 to 6 weeks of CPAP treatment [61]. OSA patients exhibited a diminished response to hypoxia, but this reverted to normal after CPAP treatment [61]. In another study [62], TCD was employed to evaluate the effects of hypoxia and hypercapnia on cerebral circulation in 20 OSA patients compared with 20 healthy subjects. Vascular response to hypoxia and hypercapnia was reduced in OSA patients, while CPAP treatment led to improvement [62]. Morgan et al. [63] have demonstrated impaired cerebrovascular responsiveness in patients with sleepdisordered breathing. This prior experience notwithstanding, it should be noted that this study included recently diagnosed OSA patients, in whom intracranial blood flow may not yet have been compromised.

Finally, we found no correlation between OSA and ABI. To the best of our knowledge, this association has not been examined. Kumagai et al. [64] have used a similar index, the cardio-ankle vascular index (CAVI), and found that it was correlated with IMT $(r=0.487, p<0.001)$. The absence of any association in our study may, at least partly, be ascribed to the small patient series. Alternatively, new-onset OSA may only be linked with early carotid lesions (as manifested in IMT) but not $\mathrm{PAD} /$ generalised atherosclerosis (as manifested by ABI). More studies are needed.

The strengths of this study are the careful evaluation of vascular disease and the inclusion of OSA patients without other known comorbidities, so confounding CVD risk factors were excluded. The limitations are the small patient series and the absence of follow-up. However, we specifically aimed to detect early atherosclerotic lesions in newly diagnosed OSA, and long-term evaluation was beyond the scope of this work.

The clinical implications of this study are that subjects with newly diagnosed OSA without known comorbidities may already exhibit increased CCA-IMT. Although further confirmation and clarification of the underlying mechanisms are desirable, this finding suggests that such subjects need to start regular assessment of carotid pathology. It is also plausible that this assessment should be coupled with patient education about vascular risk factors, appropriate lifestyle modification, and follow-up for early detection of any lesions in other vascular beds, in line with the growing appreciation of the vascular risks posed by OSA [65-67].

Our preliminary findings suggest that subjects with newly diagnosed OSA without known comorbidities exhibit a non-significant increase in CCA-IMT. They have no further evidence of vascular disease affecting the intracranial and the lower-extremity arteries. This knowledge may be seen in the context of improving diagnosis and management of carotid disease, as practised nowadays [68-70]. Additional experience is needed in a larger series.

\section{ACKNOWLEDGEMENTS}

Georgios Tsivgoulis has been supported by European Regional Development Fund -Project FNUSA-ICRC (No. CZ.1.05/1.1.00/02.0123).

\section{FUNDING}

None

\section{CONFLICTS OF INTEREST}

The authors confirm that this article content has no conflicts of interest. 


\section{REFERENCES}

[1] Shahar E, Whitney CW, Redline S, et al. Sleep-disordered breathing and cardiovascular disease: cross-sectional results of the Sleep Heart Health Study. Am J Respir Crit Care Med 2001; 163: 19-25.

[2] Peker Y, Hedner J, Norum J, Kraiczi H, Carlson J. Increased incidence of cardiovascular disease in middle-aged men with obstructive sleep apnea: a 7-year follow-up. Am J Respir Crit Care Med 2002; 166: 159-65.

[3] Katsiki N, Athyros VG, Karagiannis A, Mikhailidis DP. Characteristics other than the diagnostic criteria associated with metabolic syndrome: an overview. Curr Vasc Pharmacol 2013; [Epub ahead of print].

[4] Kostapanos MS, Elisaf MS, Mikhailidis DP. Obstructive sleep apnea and cardiovascular risk: is metabolic syndrome the link? Angiology 2012; 63: 569-73.

[5] Bassetti CL, Milanova M, Gugger M. Sleep-disordered breathing and acute ischemic stroke: diagnosis, risk factors, treatment, evolution, and long-term clinical outcome. Stroke 2006; 37: 96772.

[6] Dean RT, Wilcox I. Possible atherogenic effects of hypoxia during obstructive sleep apnea. Sleep 1993; 16(8 Suppl): S15-21.

[7] Shamsuzzaman AS, Gersh BJ, Somers VK. Obstructive sleep apnea: implications for cardiac and vascular disease. JAMA 2003; 290: 1906-14

[8] Eisensehr I, Ehrenberg BL, Noachtar S, et al. Platelet activation, epinephrine, and blood pressure in obstructive sleep apnea syndrome. Neurology 1998; 51: 188-95

[9] O'Leary DH, Polak JF. Intima-media thickness: a tool for atherosclerosis imaging and event prediction. Am J Cardiol 2002; 90: 18L-21L.

[10] Rosfors S, Hallerstam S, Jensen-Urstad K, Zetterling M, Carlstrom C. Relationship between intima-media thickness in the common carotid artery and atherosclerosis in the carotid bifurcation. Stroke 1998; 29: 1378-82.

[11] Tsivgoulis G, Vemmos K, Papamichael, C et al. Common carotid artery intima-media thickness and the risk of stroke recurrence. Stroke 2006; 37: 1913-6.

[12] O'Leary DH, Polak JF, Kronmal RA, Manolio TA, Burke GL, Wolfson SK, Jr. Carotid-artery intima and media thickness as a risk factor for myocardial infarction and stroke in older adults. Cardiovascular Health Study Collaborative Research Group. N Engl J Med 1999; 340: 14-22.

[13] Schulz R, Seeger W, Fegbeutel C, et al. Changes in extracranial arteries in obstructive sleep apnoea. Eur Respir J 2005; 25: 69-74.

[14] Suzuki T, Nakano H, Maekawa J, et al. Obstructive sleep apnea and carotid-artery intima-media thickness. Sleep 2004; 27: 129-33.

[15] Altin R, Ozdemir H, Mahmutyazicioglu K, et al. Evaluation of carotid artery wall thickness with high-resolution sonography in obstructive sleep apnea syndrome. J Clin Ultrasound 2005; 33: 806.

[16] Wattanakit K, Boland L, Punjabi NM, Shahar E. Relation of sleepdisordered breathing to carotid plaque and intima-media thickness. Atherosclerosis 2008; 197: 125-31.

[17] Alexandrov AV, Sloan MA, Tegeler CH, et al. Practice Standards for Transcranial Doppler (TCD) Ultrasound. Part II. Clinical Indications and Expected Outcomes. J Neuroimaging 2012; 22: 215-24.

[18] Klein S, Hage JJ. Measurement, calculation, and normal range of the ankle-arm index: a bibliometric analysis and recommendation for standardization. Ann Vasc Surg 2006; 20: 282-92.

[19] Stoffers J, Kaiser V, Kester A, Schouten H, Knottnerus A. Peripheral arterial occlusive disease in general practice: the reproducibility of the ankle-arm systolic pressure ratio. Scand J Prim Health Care 1991; 9: 109-14.

[20] Papanas N, Steiropoulos P, Nena E, et al. Predictors of obstructive sleep apnea in males with metabolic syndrome. Vasc Health Risk Manag 2010; 6: 281-6.

[21] Tsara V, Serasli E, Amfilochiou A, Constantinidis T, Christaki P. Greek version of the Epworth Sleepiness Scale. Sleep Breath 2004; 8: 91-5.

[22] Johns MW. A new method for measuring daytime sleepiness: the Epworth sleepiness scale. Sleep 1991; 14: 540-5.

[23] American Diabetes Association. Diagnosis and classification of diabetes mellitus. Diabetes Care 2012; 35(Suppl 1): S64-S71.
Chobanian AV, Bakris GL, Black HR, et al. The Seventh Report of the Joint National Committee on Prevention, Detection, Evaluation, and Treatment of High Blood Pressure: the JNC 7 report. JAMA 2003; 289: 2560-72.

[25] Heliopoulos I, Papaoiakim M, Tsivgoulis G, et al. Common carotid intima media thickness as a marker of clinical severity in patients with symptomatic extracranial carotid artery stenosis. Clin Neurol Neurosurg 2009; 111: 246-50.

[26] Veller MG, Fisher CM, Nicolaides AN, et al. Measurement of the ultrasonic intima-media complex thickness in normal subjects. J Vasc Surg 1993; 17: 719-25.

[27] Tsivgoulis G, Bogiatzi C, Heliopoulos I, et al. Low Ankle-Brachial Index predicts early risk of recurrent stroke in patients with acute cerebral ischemia. Atherosclerosis 2012; 220: 407-12

[28] Hirsch AT, Haskal ZJ, Hertzer NR, et al. ACC/AHA 2005 Practice Guidelines for the management of patients with peripheral arterial disease (lower extremity, renal, mesenteric, and abdominal aortic): a collaborative report from the American Association for Vascular Surgery/Society for Vascular Surgery, Society for Cardiovascular Angiography and Interventions, Society for Vascular Medicine and Biology, Society of Interventional Radiology, and the ACC/AHA Task Force on Practice Guidelines (Writing Committee to Develop Guidelines for the Management of Patients With Peripheral Arterial Disease): endorsed by the American Association of Cardiovascular and Pulmonary Rehabilitation; National Heart, Lung, and Blood Institute; Society for Vascular Nursing; TransAtlantic Inter-Society Consensus; and Vascular Disease Foundation. Circulation 2006; 113: e463-54.

[29] Steiropoulos P, Papanas N, Nena E, et al. Inflammatory markers in middle-aged obese subjects: does obstructive sleep apnea syndrome play a role? Mediators Inflamm 2010; 2010: 675320.

[30] Iber C, Ancoli-Israel S, Chesson AL, Quan SF. The AASM Manual for the Scoring of Sleep and Associated Events: Rules, Terminology, and Technical Specifications. Westchester, Ill, USA: ASM 2007.

[31] Sleep-related breathing disorders in adults: recommendations for syndrome definition and measurement techniques in clinical research. The Report of an American Academy of Sleep Medicine Task Force. Sleep 1999; 22: 667-89.

[32] Tsivgoulis G, Sharma VK, Lao AY, Malkoff MD, Alexandrov AV. Validation of transcranial Doppler with computed tomography angiography in acute cerebral ischemia. Stroke 2007; 38: 1245-9.

[33] Tsivgoulis G, Sharma VK, Hoover SL, et al. Applications and advantages of power motion-mode Doppler in acute posterior circulation cerebral ischemia. Stroke 2008; 39: 1197-204.

[34] Tsivgoulis G, Man BL, Lao AY, et al. A spectrum of knock-type Doppler signals in the intracranial vessels. Stroke 2009; 40: 644-7.

[35] Feldmann E, Wilterdink JL, Kosinski A, et al. The Stroke Outcomes and Neuroimaging of Intracranial Atherosclerosis (SONIA) trial. Neurology 2007; 68: 2099-106.

[36] Kaynak D, Goksan B, Kaynak H, Degirmenci N, Daglioglu S. Is there a link between the severity of sleep-disordered breathing and atherosclerotic disease of the carotid arteries? Eur J Neurol 2003; 10: 487-93.

[37] Gamero L, Levenson J, Armentano R, et al. Carotid wall inertial index increase is related to intima-media thickening in hypertensive patients. J Hypertens 1999; 17: 1825-9.

[38] Hodis HN, Mack WJ, LaBree L, et al. Reduction in carotid arterial wall thickness using lovastatin and dietary therapy: a randomized controlled clinical trial. Ann Intern Med 1996; 124: 548-56.

[39] De Pergola G, Ciccone M, Pannacciulli N, et al. Lower insulin sensitivity as an independent risk factor for carotid wall thickening in normotensive, non-diabetic, non-smoking normal weight and obese premenopausal women. Int J Obes Relat Metab Disord 2000; 24: 825-9.

[40] Szaboova E, Tomori Z, Donic V, Petrovicova J, Szabo P. Sleep apnoea inducing hypoxemia is associated with early signs of carotid atherosclerosis in males. Respir Physiol Neurobiol 2007; 155: $121-7$.

[41] Baguet JP, Hammer L, Levy P, et al. The severity of oxygen desaturation is predictive of carotid wall thickening and plaque occurrence. Chest 2005; 128: 3407-12.

[42] Li C, Zhang XL, Liu H, Wang ZG, Yin KS. Association among plasma interleukin-18 levels, carotid intima- media thickness and severity of obstructive sleep apnea. Chin Med J (Engl) 2009; 122: 24-9. 
[43] Minoguchi K, Yokoe T, Tazaki T, et al. Increased carotid intimamedia thickness and serum inflammatory markers in obstructive sleep apnea. Am J Respir Crit Care Med 2005; 172: 625-30.

[44] Saletu M, Nosiska D, Kapfhammer G, et al. Structural and serum surrogate markers of cerebrovascular disease in obstructive sleep apnea (OSA): association of mild OSA with early atherosclerosis. J Neurol 2006; 253: 746-52.

[45] Steiropoulos P, Papanas N, Nena E, et al. Mean Platelet Volume and Platelet Distribution Width in patients with Chronic Obstructive Pulmonary Disease: the role of comorbidities. Angiology 2012. [Epub ahead of print]

[46] Saletu M, Sauter C, Lalouschek W, et al. Is excessive daytime sleepiness a predictor of carotid atherosclerosis in sleep apnea? Atherosclerosis 2008; 196: 810-6

[47] Nena E, Steiropoulos P, Papanas N, et al. Sleepiness as a marker of glucose deregulation in obstructive sleep apnea. Sleep Breath 2012; 16:181-6.

[48] Monneret D, Pepin JL, Godin-Ribuot D, et al. Association of urinary 15-F2t-isoprostane level with oxygen desaturation and carotid intima-media thickness in nonobese sleep apnea patients. Free Radic Biol Med 2010; 48: 619-25.

[49] Jung CH, Yu JH, Bae SJ, et al. Serum gamma-glutamyltransferase is associated with arterial stiffness in healthy individuals. Clin Endocrinol (Oxf) 2011; 75: 328-34.

[50] Kazemi-Shirazi L, Endler G, Winkler S, Schickbauer T, Wagner O, Marsik C. Gamma glutamyltransferase and long-term survival: is it just the liver? Clin Chem 2007; 53: 940-6.

[51] Wannamethee SG, Lennon L, Shaper AG. The value of gammaglutamyltransferase in cardiovascular risk prediction in men without diagnosed cardiovascular disease or diabetes. Atherosclerosis 2008; 201: 168-75.

[52] Mason JE, Starke RD, Van Kirk JE. Gamma-glutamyl transferase: a novel cardiovascular risk biomarker. Prev Cardiol 2010; 13: 3641 .

[53] Shimizu Y, Imano H, Ohira T, et al. gamma-Glutamyltranseptidase and incident stroke among Japanese men and women: the Circulatory Risk in Communities Study (CIRCS). Stroke 2010; 41: 385-8.

[54] Kanbay A, Kaya E, Buyukoglan H, et al. Serum gamma-glutamyl transferase activity is an independent predictor for cardiovascular disease in obstructive sleep apnea syndrome. Respir Med 2011; 105: 637-42.

[55] Zureik M, Kony S, Neukirch C, et al. Bronchial hyperresponsiveness to methacholine is associated with increased common carotid intima-media thickness in men. Arterioscler Thromb Vasc Biol 2004; 24: 1098-103.

[56] Ebrahim S, Papacosta O, Whincup P, et al. Carotid plaque, intima media thickness, cardiovascular risk factors, and prevalent cardiovascular disease in men and women: the British Regional Heart Study. Stroke 1999; 30: 841-50.

[57] Wannamethee SG, Shaper AG, Ebrahim S. Respiratory function and risk of stroke. Stroke 1995; 26: 2004-10.

[58] Truelsen T, Prescott E, Lange P, Schnohr P, Boysen G. Lung function and risk of fatal and non-fatal stroke. The Copenhagen City Heart Study. Int J Epidemiol 2001; 30: 145-51.

[59] Guo X, Pantoni L, Simoni M, et al. Midlife respiratory function related to white matter lesions and lacunar infarcts in late life: the Prospective Population Study of Women in Gothenburg, Sweden. Stroke 2006; 37: 1658-62.

[60] Liao D, Higgins M, Bryan NR, et al. Lower pulmonary function and cerebral subclinical abnormalities detected by MRI: the Atherosclerosis Risk in Communities study. Chest 1999; 116: 1506.

[61] Foster GE, Hanly PJ, Ostrowski M, Poulin MJ. Effects of continuous positive airway pressure on cerebral vascular response to hypoxia in patients with obstructive sleep apnea. Am J Respir Crit Care Med 2007; 175: 720-5.

[62] Reichmuth KJ, Dopp JM, Barczi SR, et al. Impaired vascular regulation in patients with obstructive sleep apnea: effects of continuous positive airway pressure treatment. Am J Respir Crit Care Med 2009; 180: 1143-50.

[63] Morgan BJ, Reichmuth KJ, Peppard PE, et al. Effects of sleepdisordered breathing on cerebrovascular regulation: A populationbased study. Am J Respir Crit Care Med 2010; 182: 1445-52.

[64] Kumagai T, Kasai T, Kato M, et al. Establishment of the cardioankle vascular index in patients with obstructive sleep apnea. Chest 2009; 136: 779-86.

[65] Kostapanos MS, Mikhailidis DP, Elisaf MS, Steiropoulos P, Papanas N. Obstructive sleep apnoea syndrome and cardiovascular risk. Arch Med Sci 2012; 8: 1115-6.

[66] Nena E, Papanas N, Steiropoulos P, et al. Mean Platelet Volume and Platelet Distribution Width in non-diabetic subjects with obstructive sleep apnoea syndrome: new indices of severity? Platelets 2012; 23: 447-54.

[67] Athyros VG, Tziomalos K, Karagiannis A, Mikhailidis DP Cardiovascular benefits of bariatric surgery in morbidly obese patients. Obes Rev 2011; 12: 515-24.

[68] Paraskevas KI, Mikhailidis DP, Moore WS, Veith FJ. Optimal contemporary management of symptomatic and asymptomatic carotid artery stenosis. Vascular 2011; 19: 117-20.

[69] Abbott AL, Adelman MA, Alexandrov AV, et al. Why the US Center for Medicare and Medicaid Services should not extend reimbursement indications for carotid artery angioplasty/stenting. Angiology 2012; 63: 639-44.

[70] Katsiki N, Papadopoulou SK, Fachantidou AI, Mikhailidis DP. Smoking and vascular risk: are all forms of smoking harmful to all types of vascular disease? Public Health, 2013; 127(5): 435-41.

Received: June 05, 2013

Accepted: June 05, 2013

(C) Steiropoulos et al.; Licensee Bentham Open.

This is an open access article licensed under the terms of the Creative Commons Attribution Non-Commercial License (http://creativecommons.org/licenses/ by-nc/3.0/) which permits unrestricted, non-commercial use, distribution and reproduction in any medium, provided the work is properly cited. 